\title{
DEEP SPITZER SPECTROSCOPY OF THE "FLYING SAUCER” EDGE-ON DISK: LARGE GRAINS BEYOND 50 AU
}

\author{
Klaus M. Pontoppidan, ${ }^{1,2}$ Karl R. Stapelfeldt, ${ }^{3}$ Geoffrey A. Blake, ${ }^{1}$ \\ Ewine F. van Dishoeck, ${ }^{4}$ And Cornelis P. Dullemond ${ }^{5}$ \\ Received 2006 December 11; accepted 2007 February 15; published 2007 February 27
}

\begin{abstract}
We present deep Spitzer IRS low-resolution $(\lambda / \Delta \lambda \sim 100)$ 5-35 $\mu \mathrm{m}$ spectroscopy of the edge-on disk the "Flying Saucer" (2MASS J16281370-2431391) in the Ophiuchus molecular cloud. The SED exhibits the characteristic two-peak shape predicted for a circumstellar disk viewed very close to edge-on. The short-wavelength peak is entirely due to photons scattered off the surface of the disk, while the long-wavelength peak is due to thermal emission from the disk itself. The Spitzer spectrum represents the first spectroscopic detection of scattered light out to $15 \mu \mathrm{m}$ from a bona fide, isolated edge-on disk around a T Tauri star. The depth and the wavelength of the mid-infrared "valley" of the SED give direct constraints on the size distribution of large grains in the disk. Using a 2D continuum radiative transfer model, we find that a significant amount of 5-10 $\mu \mathrm{m}$-sized grains is required in the surface layers of the disk at radii of 50-300 AU. The detection of relatively large grains in the upper layers implies that vertical mixing is effective, since grain growth models predict that the grains would otherwise settle deep in the disk on short timescales. Additionally, we tentatively detect the $9.66 \mu \mathrm{m} S(3)$ line of $\mathrm{H}_{2}$ and the $11.2 \mu \mathrm{m}$ emission feature due to PAHs.
\end{abstract}

Subject headings: circumstellar matter — infrared: ISM — planetary systems: protoplanetary disks stars: individual (2MASS J16281370-2431391) — stars: pre-main-sequence

Online material: color figures

\section{INTRODUCTION}

Highly inclined circumstellar disks are particularly well suited for studies of the dust properties in optically thick protoplanetary systems. The very high degree of obscuration toward the bright inner parts of these disks acts as a natural coronagraph and thereby increases the contrast to the fainter outer parts by several orders of magnitude, enabling direct studies of the optical and infrared properties of the disk surface layers at radii of $\geq 50 \mathrm{AU}$ (the region of the $\tau=1$ surface to scattering as seen by an observer). In particular, the scattering properties, and therefore the grain size distributions and the vertical height of the disk, can be constrained using spatially resolved imaging. Examples of such studies employing optical and near-infrared scattered light images of edge-on disks are found in Burrows et al. (1996), Stapelfeldt et al. (1998), Cotera et al. (2001), Stapelfeldt et al. (2003), and Wolf et al. (2003).

These studies have shown that the extended emission at optical and near-infrared wavelengths has a morphology closely resembling that theoretically expected for edge-on disks, i.e., two flattened reflection nebulosities bisected by a dark lane in the disk plane. Attempts to use these relatively short wavelengths to search for grains larger than those found in the interstellar medium have produced somewhat inconclusive results, and it has become clear that observations at longer wavelengths are required (e.g., Brandner et al. 2000; Wood et al. 2002). A number of mid-infrared images have been made of edge-on disks. Spatially resolved emission at $11.8 \mu \mathrm{m}$ from the edge-on disk HK Tau B was presented by McCabe et al. (2003), who used the image to infer a grain size distribution with relatively large grains $(1.5-3.0 \mu \mathrm{m})$. Perrin et al.

\footnotetext{
${ }^{1}$ California Institute of Technology, Division of Geological and Planetary Sciences, Pasadena, CA 91125; pontoppi@gps.caltech.edu, gab@gps.caltech.edu.

${ }^{2}$ Hubble Fellow.

3 Jet Propulsion Laboratory, Pasadena, CA 91109; krs@exoplanet.jpl.nasa.gov.

${ }^{4}$ Leiden Observatory, NL-2300 RA Leiden, Netherlands; ewine@strw .leidenuniv.nl.

${ }^{5}$ Max-Planck-Institut für Astronomie, D69117 Heidelberg, Germany; dullemon@ mpia-hd.mpg.de.
}

(2006) presented mid-infrared images of an edge-on disk clearly dominated by polycyclic aromatic hydrocarbon (PAH) emission rather than scattered light.

A problem is that edge-on disks tend to be very faint in the mid-infrared, requiring very sensitive observational facilities. Highly sensitive spectroscopy offered by the Spitzer Space Telescope provides new possibilities. Spitzer will not spatially resolve the mid-infrared emission, except for extremely large disks, but can obtain spectroscopic information that provides essential constraints for systems that have already been imaged at shorter wavelengths. One important advantage of spectroscopy is that line emission can be distinguished from scattered light, a distinction that becomes essential when trying to constrain scattering properties of the dust.

In this Letter, we report deep mid-infrared spectroscopic observations using the Infrared Spectrograph (IRS) on Spitzer of the "Flying Saucer" edge-on disk located on the outskirts of the Ophiuchus star-forming cloud core, first identified in highresolution near-infrared images (Grosso et al. 2003). The spectrum was obtained as part of the "From Cores to Disks" (c2d) Legacy program (Evans et al. 2003). The Flying Saucer is one of the most isolated edge-on disks known with a foreground visual extinction less than a few magnitudes, and it is viewed almost exactly edge-on $\left(i \gtrsim 85^{\circ}\right)$.

\section{DATA REDUCTION AND RESULTS}

The Flying Saucer (2MASS J16281370-2431391; $\alpha=$ $16^{\mathrm{h}} 28^{\mathrm{m}} 13.7^{\mathrm{h}}, \delta=-24^{\circ} 31^{\prime} 39^{\prime \prime}$ [J2000]) was observed using both the Short-Low (SL2 $=5.2-7.5 \mu \mathrm{m}, \mathrm{SL} 1=7.5-14.5 \mu \mathrm{m})$ and Long-Low (LL2 $=14.2-21.0 \mu \mathrm{m}, \mathrm{LL} 1=19.8-38.1 \mu \mathrm{m})$ modules of the IRS (AOR key 0009829632). Each SL order was observed for $10 \times 61 \mathrm{~s}$, and each LL order for $20 \times$ $31.5 \mathrm{~s}$. The spectral extraction was performed from the Basic Calibrated Data pipeline (ver. S13.2.0) products by co-adding each nod position and then subtracting the two resulting twodimensional (2D) spectra to effectively remove the background. 
TABLE 1

Mid-Infrared Photometry of THE FLYING SAUCER

\begin{tabular}{cccc}
\hline \hline $\begin{array}{c}\text { Wavelength } \\
(\mu \mathrm{m})\end{array}$ & $\begin{array}{c}\text { Flux } \\
(\mathrm{mJy})\end{array}$ & Ref. \\
\hline $3.6 \ldots \ldots \ldots$ & $2.43 \pm 0.24$ & $\mathrm{c} 2 \mathrm{~d}$ \\
$4.5 \ldots \ldots \ldots$ & $2.32 \pm 0.23$ & $\mathrm{c} 2 \mathrm{~d}$ \\
$5.7 \ldots \ldots \ldots$ & $1.70 \pm 0.17$ & $\mathrm{c} 2 \mathrm{~d}$ \\
$7.9 \ldots \ldots \ldots$ & $0.84 \pm 0.08$ & $\mathrm{c} 2 \mathrm{~d}$ \\
$24 \ldots \ldots \ldots$. & $2.0 \pm 0.5$ & $\mathrm{c} 2 \mathrm{~d}$ \\
$70 \ldots \ldots \ldots$ & $<300$ & $\mathrm{c} 2 \mathrm{~d}$ \\
\hline
\end{tabular}

Due to the presence of a bright source on the peak-up array for the SL1 observation, the noise at wavelengths between 12.7 and $14.5 \mu \mathrm{m}$ is significantly higher than in other parts of the spectrum. The Flying Saucer is clearly detected in SL1 and SL2, as well as in LL1; there is no detection in LL2, consistent with a location of the flux minimum around $20 \mu \mathrm{m}$. One-dimensional spectra were extracted using 3 pixel $\left(5.4^{\prime \prime}\right)$ apertures to optimize the signal-to-noise ratio $(\mathrm{S} / \mathrm{N})$. To aid the modeling efforts, Infrared Array Camera (IRAC) and Multiband Imaging Photometer for Spitzer (MIPS) photometry has been extracted from the c2d photometric database. The spectra match the IRAC band 3 $(5.7 \mu \mathrm{m})$ and band $4(7.9 \mu \mathrm{m})$ photometry within the accuracy of the absolute calibration. The difference in slope between the spectrum and IRAC 3 and IRAC4 may be due to the source being extended; the SL slit width is 3.6". The source is detected in MIPS1 $(24 \mu \mathrm{m})$, and only an upper limit is available in MIPS2 $(70 \mu \mathrm{m})$ (see Table 1).

The resulting SL spectrum is shown in Figure 1 and shows two weak, narrow emission features at $\sim 9.7$ and $\sim 11.2 \mu$ m with line-to-continuum ratios of $\sim 1$. Care should be taken in interpreting these features because the behavior of the spectrograph is not yet fully understood, and features at the 5\%-10\% level may be spurious. Assuming Gaussian noise, the two emission features are detected with S/Ns of 4-5. Inspection of the coadded 2D spectra confirms that the features are compact and centered on the continuum position, at least along the slit, which is aligned at a position angle of $5^{\circ}$, or roughly perpendicular to the disk plane. We tentatively identify the emission features as the $\mathrm{H}_{2} S(3)$ line at $9.66 \mu \mathrm{m}$ and the out-of-plane bending mode of PAHs at $11.2 \mu \mathrm{m}$. The strength of the $S(3)$ line corresponds to an integrated flux of $(9 \pm 2) \times 10^{-15} \mathrm{ergs} \mathrm{s}^{-1} \mathrm{~cm}^{-2}$, while the $11.2 \mu \mathrm{m}$ line flux is $(15 \pm 3) \times 10^{-15} \mathrm{ergs} \mathrm{s}^{-1} \mathrm{~cm}^{-2}$, using a first-order polynomial fit to the continuum. For an excitation temperature of $\sim 1000 \mathrm{~K}$, the other $\mathrm{H}_{2}$ lines are expected to be below the detection limit, taking into account the higher noise at the $6.9 \mu \mathrm{m} S(5)$ line. The PAH line flux is $2-3$ orders of magnitude less, and the line-to-continuum ratio is about 10 times larger than those determined for other PAH-emitting disks in the c2d sample (Geers et al. 2006).

The spectral energy distribution (SED), shown in Figure 2, declines between 4 and $14 \mu \mathrm{m}$. The LL spectrum falls below the detection limit of $\sim 1 \mathrm{mJy}$ below $24 \mu \mathrm{m}$ but rises steeply at longer wavelengths. This spectral shape closely resembles that theoretically expected for a circumstellar disk viewed close to edge-on (see, e.g., Whitney et al. 2003). The observed midinfrared fluxes of $1 \mathrm{mJy}$ match closely those predicted by the Grosso et al. (2003) model. At short wavelengths $(\lambda \lesssim 40 \mu \mathrm{m})$, the optical depth to the source (the star and hot dust in the inner disk) is much greater than unity. This means that all of the short-wavelength photons are those that are scattered off the surface of the disk at large radii-in this case, 50-300 AU. The SED of the scattered light will have a shape that can be

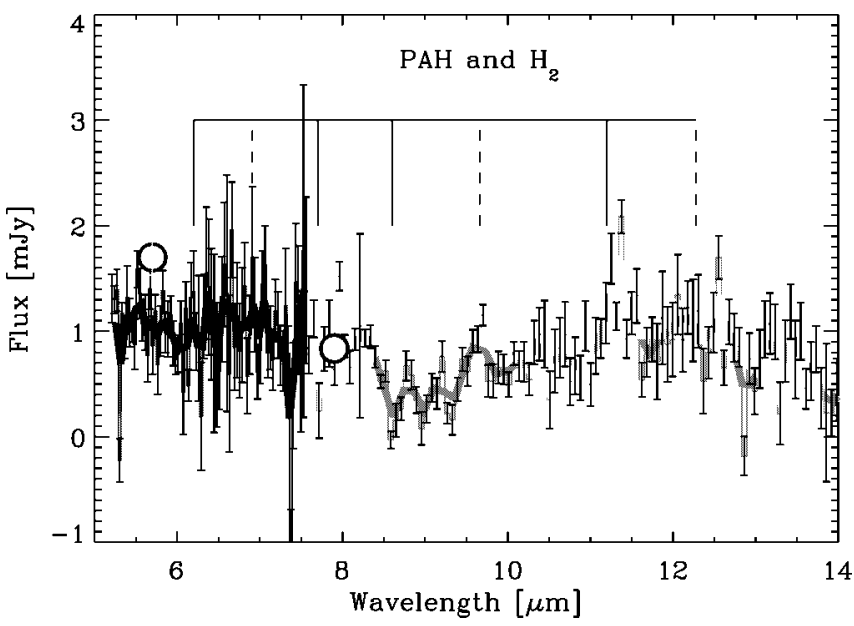

FIG. 1.-Short-Low spectrum showing the tentative emission features at 9.7 and $11.2 \mu \mathrm{m}$. The vertical bars show the location of common PAH features (solid bars) as well as the $S(2), S(3)$, and $S(4)$ lines of molecular hydrogen (dashed bars). The filled circles show the IRAC photometry in bands 3 and 4 . The thick curve shows a spectrum that has been smoothed with a two-point boxcar filter. [See the electronic edition of the Journal for a color version of this figure.]

calculated roughly as the spectrum of the inner star+disk system multiplied by the albedo of the dust in the outer disk. At long wavelengths, the SED is dominated by thermal emission from the cold, outer part of the disk. These two components create a characteristic double-peaked SED with a minimum in the range 5-20 $\mu \mathrm{m}$ that is unrelated to, but may in some cases be confused with, the silicate band at $9.7 \mu \mathrm{m}$.

\section{CONSTRAINING THE DUST SIZE DISTRIBUTION}

We have constructed a $2 \mathrm{D}$ continuum radiative transfer model of the Flying Saucer with a setup similar to that of Pontoppidan et al. (2005) using the Monte Carlo code RADMC (Dullemond \& Dominik 2004). This setup uses an axisymmetric density structure on a polar grid to calculate images and spectra. We use Mie theory to calculate the input opacities to

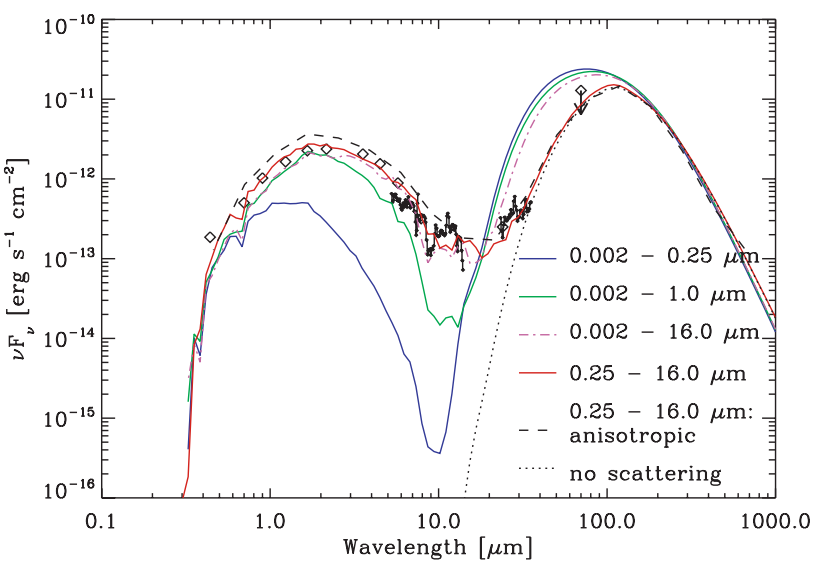

FIG. 2.-Model fit to the SED of the Flying Saucer. The optical and nearinfrared points are taken from Grosso et al. (2003). Grain size distributions weighted toward increasing grain sizes move the mid-infrared minimum in the SED to progressively longer wavelengths. The dashed curve is the same as the best-fit model (red curve), but with a proper implementation of anisotropic scattering. The dotted curve shows the SED with the scattered photons removed. No photons directly from the central star are seen at any wavelength. The colors of the curves refer to the same grain size distributions as those in Fig. 3. 

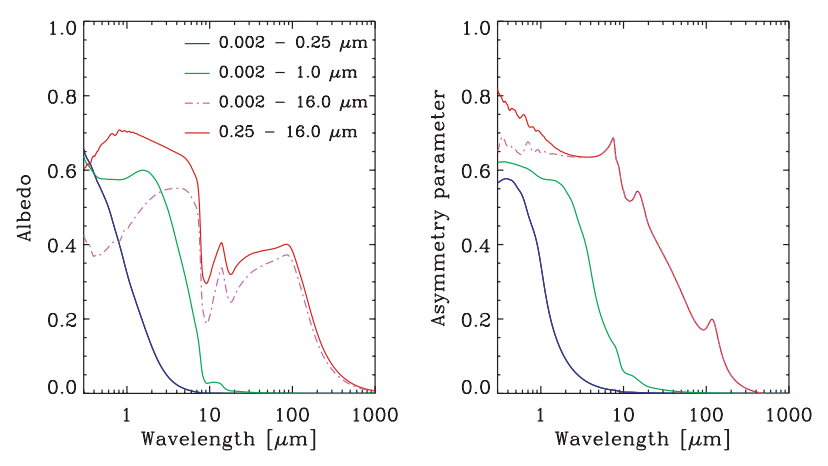

FIG. 3.-Albedos and the angle-averaged phase functions or "asymmetry parameters" ( $\langle\cos \theta\rangle=g$ ) for different grain size distributions. In principle, the albedo is a measure of the ability of a grain population to scatter light into the line of sight without removing it through absorption and reemission at longer wavelengths.

the model and explore a range of different grain size distributions. These are modeled by a power law size distribution $d n(a) / d a \propto a^{-\alpha}$ with minimum and maximum grain radii, $a_{\min }$ and $a_{\max }$, and $\alpha=3.5$. The dust material is silicate with optical constants from Draine \& Lee (1984) and amorphous carbon (800 K experiment of Jager et al. 1998). For simplicity, it is assumed that carbon and silicate grains are separate populations with $15 \%$ of the mass in carbon. Figure 3 shows the scattering properties for some of the grain models.

We model the physical disk structure using the Grosso et al. (2003) model parameters as a starting point. These include an outer disk scale height of $H(100 \mathrm{AU}) / 100 \mathrm{AU}=0.15$, a disk mass of $2 \times 10^{-3} M_{\odot}$, a surface density power law $\Sigma(R) \propto$ $R^{-1}$, and a flaring scale height that varies as $h \propto R^{5 / 4}$. The disk has a radius of $300 \mathrm{AU}$. The disk parameters have been obtained by Grosso et al. (2003) by detailed fitting to the near-infrared images, using a grain size distribution with a maximum grain size of $\sim 50 \mu \mathrm{m}$. Since no photometry is available above $70 \mu \mathrm{m}$, the properties of the disk midplane are not well constrained. Some changes to the Grosso et al. (2003) model were made. First, we adopted a slightly smaller distance of $125 \mathrm{pc}$ instead of $140 \mathrm{pc}$ (de Geus et al. 1989). Second, to improve the fit to the nearinfrared and IRAC photometry, we found it necessary to include a relative accretion luminosity of $L_{\text {acc }} / L_{*}=0.6$ to the system, modeled by adding a blackbody with a temperature of $1400 \mathrm{~K}$ to the input stellar spectrum, which is modeled by a $0.084 L_{\odot}$, $3500 \mathrm{~K}$ stellar atmosphere from Kurucz (1979), as well as setting the foreground extinction to $A_{V}=0.5 \mathrm{mag}$. The accretion luminosity $A_{V}$ and effective temperature are somewhat interdependent, so other combinations may be possible; in particular, the central star may be cooler and more luminous, requiring less accretion. Removing the accretion component entirely will lower the 1$5 \mu \mathrm{m}$ flux by a factor of $\sim 2$. In any case, these parameters do not affect wavelengths longer than $\sim 5 \mu \mathrm{m}$, and therefore they will not affect the derived grain size distribution.

Given a set of parameters determining the physical structure of the disk, the grain size distribution strongly affects the shape and the location of the minimum of the SED in the mid-infrared. In Figure 2, models with different grain size distributions are compared to the observed SED and, in particular, to the IRS spectrum. As larger grains are included in the grain population, the mid-infrared flux due to scattered photons increases relative to the rest of the SED. While light is always scattered at the surface, where the scattering optical depth $\left(\tau_{\text {sca }}\right)$ is unity, the albedo controls how much light actually survives (is not absorbed

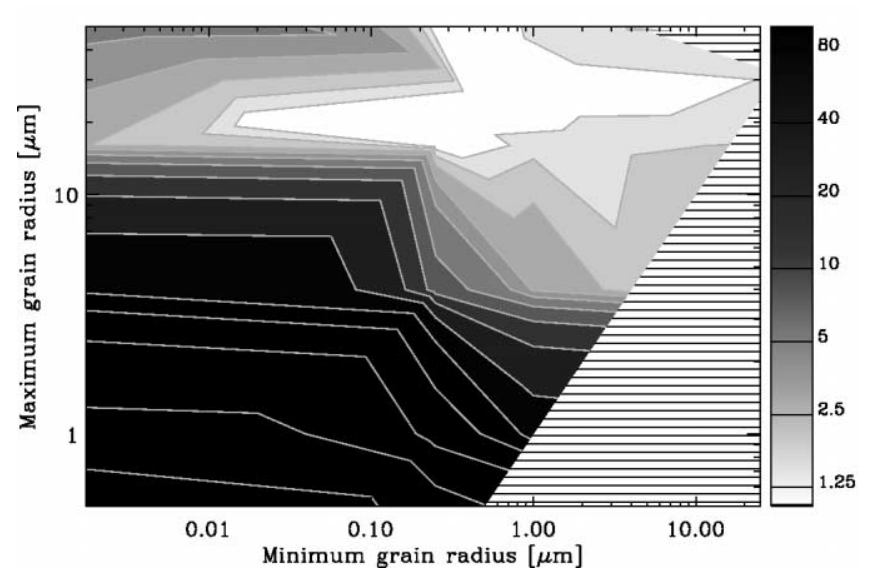

FIG. 4.-Goodness-of-fit surface for the calculated grid of models. The contours have been normalized such that the best-fitting model has a goodness of fit of unity. The contours shown are in steps of $40 \%$, the lowest having a value of 1.4. The hatched pattern indicates the area where no reasonable models can been calculated because $a_{\min }>a_{\max }$. [See the electronic edition of the Journal for a color version of this figure.]

and reemitted at longer wavelengths) to be scattered at $\tau_{\text {sca }}=$ 1. According to Figure 3, a significant population of $\sim 10 \mu \mathrm{m}$ grains is required in order to have an albedo $=Q_{\text {sca }} /\left(Q_{\text {abs }}+\right.$ $\left.Q_{\text {sca }}\right) \gtrsim 0.1$ at $20 \mu \mathrm{m}$. Additionally, as the small grains are removed, the thermal emission peak shifts to longer wavelengths.

We ran a grid of models, varying the minimum and maximum grain sizes, while keeping the density structure constant, to minimize the quantity $\int\left(F_{\nu \text {, model }}(\nu)-F_{\nu \text {, obs }}(\nu)\right)^{2} d \nu$. Figure 4 shows the goodness-of-fit surface. Inspection shows that good matches to the SED are found for grain size distributions with $a_{\text {min }} \gtrsim 0.5 \mu \mathrm{m}$ and $a_{\max } \gtrsim 10 \mu \mathrm{m}$. While the exact limits to the grain size distribution are not strictly constrained from the SED alone, the presented model clearly excludes grain size distributions with no grains larger than $\sim 5 \mu \mathrm{m}$ or distributions that are dominated (in mass) by grains that are $\leqslant 0.5 \mu \mathrm{m}$. The upper cutoff for the grain size distribution may be significantly larger. The required presence of large grains is very stable to changes in the density structure of the disk and slope of the grain size distribution. Whether or not there is a lower cutoff to the grain size is less secure. For instance, a shallower grain size distribution was found to, at least in part, lessen the need for a lower cutoff of grain sizes. Additionally, a larger disk mass may be required since our best-fitting $J$-band opacity is $\sim 3$ times lower than that used by Grosso et al. (2003). Models with higher disk masses lessen the need for a lower grain size cutoff by shifting the thermal peak in the SED to longer wavelengths, but they do not affect the scattering peak. We are therefore confident that large grains are present in the Flying Saucer, while the evidence for a lack of small grains is somewhat more ambiguous. This result strengthens similar conclusions obtained for other edge-on disks using less constraining data at shorter wavelengths (Wood et al. 2002; McCabe et al. 2003) and provides strong evidence for the presence of grain growth in the outer disk of the Flying Saucer.

The model grid uses isotropic scattering, thereby requiring much fewer photon packages than if anisotropic scattering were used. While not a proper treatment of scattering of large grains, computing time is drastically cut this way. To ensure that the conclusions are not affected, we also calculated the SED of the best-fitting model using a proper anisotropic scattering model. Anisotropic scattering has the effect of making the image of the disk more compact around the central star. The effect on the 
SED is small as seen in Figure 2; it changes the flux by $~ 50 \%$ at wavelengths dominated by scattered photons. This difference is very small compared to the 2-3 orders of magnitude dependence on the grain size distribution in the mid-infrared.

\section{DISCUSSION}

The IRS spectrum of the Flying Saucer has provided important constraints on the nature of disk material in the surface layers $(H / R \sim 0.1)$ of the disk at radii larger than $50 \mathrm{AU}$ in several different ways. First, dust grains in this part of the disk are much larger than grains in the interstellar medium, clearly implying significant grain growth. Second, tentative emission features from PAHs and $\mathrm{H}_{2}$ reveal clues about emission excited by UV photon processes in the outer disk. Had the disk been viewed face-on, these faint features would have disappeared in the glare from the warm inner parts of the disk; at $10 \mu \mathrm{m}$, the Flying Saucer would be 200 times brighter if viewed face-on.

Evidence from silicate emission features has already indicated that grains have grown to several micrometers (Bouwman et al. 2001; van Boekel et al. 2005) at small disk radii, within $\sim 10 \mathrm{AU}$ (van Boekel et al. 2004), in Herbig Ae disks as well as T Tauri stars (Kessler-Silacci et al. 2006). In contrast, mid-infrared observations of scattered light such as those presented in this Letter can reveal the presence of large grains in the surface layers much farther away from the central star. While millimeter continuum studies have also found evidence of large grains in the outer regions of disks (e.g., Natta et al. 2004; Rodmann et al. 2006), such observations probe only the midplane. In the case of the Flying Saucer, we have shown that significant grain growth has taken place at large distances from the star (up to several hundred AU), consistent with the conclusions of McCabe et al. (2003) in the case of HK Tau B, a similar edge-on disk. When comparing this result to recent models of grain growth and settling (e.g., Dullemond \& Dominik 2004, 2005), an apparent problem appears. These models predict that grain growth to sizes $>10 \mu \mathrm{m}$, followed by rapid settling to the disk midplane, takes place on very short timescales $\left(10^{3}-10^{5} \mathrm{yr}\right)$. This will effectively remove larger grains from the disk surface on a timescale that increases with radius and decreases with grain size. The new observational evidence presented here shows that large grains are present in the disk surface not only close to the star, as found in other studies, but also at much larger distances. This implies that in both these regimes, there is an efficient mechanism for preventing grains in the $\sim 10 \mu \mathrm{m}$ range from settling.

Adding to the puzzle is the indication of the presence of very small grains, such as PAHs, in the same general region of the disk as the large grains. Even if the detection of PAHs in the Flying Saucer is only tentative, some edge-on disks clearly show very strong PAH features (Perrin et al. 2006). Very small grains will not settle, and so the questions are as follows: why have they not been removed by the grain coagulation producing the observed population of large grains and is there a process to replenish them?

Another possibility for explaining the presence of PAH emission is that the $11.2 \mu \mathrm{m}$ feature may be light from the inner parts of the disk scattered on the outer surface. This requires that the line-to-continuum ratio of the PAH emission from the inner disk is the same as that observed in the Flying Saucer. Observations of face-on disks have shown that such strong line emission is rare but not impossible (Geers et al. 2006).

The tentative detection of the $S(3)$ line of $\mathrm{H}_{2}$ is interesting. The $S(3)$ line dominates the $\mathrm{H}_{2}$ line spectrum for gas at a temperature of a few hundred kelvins. Detailed modeling by Nomura \& Millar (2005) has shown that, in the presence of a significant UV excess, the $S(3)$ line flux from a disk very similar to that of the Flying Saucer is expected to be $\sim 9 \times 10^{-15} \mathrm{ergs}$ $\mathrm{s}^{-1} \mathrm{~cm}^{-2}$, with most of the flux originating in the uppermost layers of the disk at radii of 10-100 AU. If confirmed, the presence of the line can be interpreted as an indicator of a strongly enhanced UV field in the upper layers.

Finally, the possible presence of high-contrast emission lines in the spectrum of the Flying Saucer suggests that edge-on disks may be excellent targets for looking for fluorescent tracers in the outer disk that would not have been seen if the disk had been viewed face-on. This illustrates the need for the kind of sensitive space-borne high-resolution mid-infrared spectroscopy in the future that can be offered by SOFIA and the James Webb Space Telescope.

We are grateful for a constructive review by an anonymous referee. K. M. P. is supported by NASA through Hubble Fellowship grant 01201.01 awarded by the Space Telescope Science Institute, which is operated by the Association of Universities for Research in Astronomy, Inc., for NASA, under contract NAS 5-26555. Support for this work, part of the Spitzer Space Telescope Legacy Science Program, was provided by NASA through contracts 1224608 and 1230779 issued by the Jet Propulsion Laboratory, California Institute of Technology, under NASA contract 1407. E. F. v. D. acknowledges an NWO Spinoza prize.

\section{REFERENCES}

Bouwman, J., Meeus, G., de Koter, A., Hony, S., Dominik, C., \& Waters, L. B. F. M. 2001, A\&A, 375, 950

Brandner, W., et al. 2000, A\&A, 364, L13

Burrows, C. J., et al. 1996, ApJ, 473, 437

Cotera, A. S., et al. 2001, ApJ, 556, 958

de Geus, E. J., de Zeeuw, P. T., \& Lub, J. 1989, A\&A, 216, 44

Draine, B. T., \& Lee, H. M. 1984, ApJ, 285, 89

Dullemond, C. P., \& Dominik, C. 2004, A\&A, 417, 159 2005, A\&A, 434, 971

Evans, N. J., et al. 2003, PASP, 115, 965

Geers, V. C., et al. 2006, A\&A, 459, 545

Grosso, N., Alves, J., Wood, K., Neuhäuser, R., Montmerle, T., \& Bjorkman, J. E. 2003, ApJ, 586, 296

Jager, C., Mutschke, H., \& Henning, T. 1998, A\&A, 332, 291

Kessler-Silacci, J., et al. 2006, ApJ, 639, 275

Kurucz, R. L. 1979, ApJS, 40, 1

McCabe, C., Duchêne, G., \& Ghez, A. M. 2003, ApJ, 588, L113

Natta, A., Testi, L., Neri, R., Shepherd, D. S., \& Wilner, D. J. 2004, A\&A, 416, 179
Nomura, H., \& Millar, T. J. 2005, A\&A, 438, 923

Perrin, M. D., Duchêne, G., Kalas, P., \& Graham, J. R. 2006, ApJ, 645, 1272 Pontoppidan, K. M., Dullemond, C. P., van Dishoeck, E. F., Blake, G. A., Boogert, A. C. A., Evans, N. J., II, Kessler-Silacci, J. E., \& Lahuis, F. 2005, ApJ, 622, 463

Rodmann, J., Henning, T., Chandler, C. J., Mundy, L. G., \& Wilner, D. J. 2006, A\&A, 446, 211

Stapelfeldt, K. R., Krist, J. E., Menard, F., Bouvier, J., Padgett, D. L., \& Burrows, C. J. 1998, ApJ, 502, L65

Stapelfeldt, K. R., Ménard, F., Watson, A. M., Krist, J. E., Dougados, C., Padgett, D. L., \& Brandner, W. 2003, ApJ, 589, 410

van Boekel, R., Min, M., Waters, L. B. F. M., de Koter, A., Dominik, C., van den Ancker, M. E., \& Bouwman, J. 2005, A\&A, 437, 189

van Boekel, R., et al. 2004, Nature, 432, 479

Whitney, B. A., Wood, K., Bjorkman, J. E., \& Wolff, M. J. 2003, ApJ, 591, 1049

Wolf, S., Padgett, D. L., \& Stapelfeldt, K. R. 2003, ApJ, 588, 373

Wood, K., Wolff, M. J., Bjorkman, J. E., \& Whitney, B. 2002, ApJ, 564, 887 